\title{
Excitation of MHD waves in magnetized anisotropic cosmologies
}

\author{
A. Kuiroukidis ${ }^{1,2}$, K. Kleidis ${ }^{1,3}$, D. B. Papadopoulos ${ }^{1}$, and L. Vlahos ${ }^{1}$ \\ 1 Department of Physics, Aristotle University of Thessaloniki, 54124 Thessaloniki, Greece \\ e-mail: kleidis@astro.auth.gr \\ 2 Department of Informatics, Technological Education Institute of Serres, 64124 Serres, Greece \\ 3 Department of Civil Engineering, Technological Education Institute of Serres, 64124 Serres, Greece
}

Received 16 January 2007 / Accepted 16 April 2007

\begin{abstract}
The excitation of cosmological perturbations in an anisotropic cosmological model and in the presence of a homogeneous magnetic field was studied, using the resistive magnetohydrodynamic (MHD) equations. We have shown that fast-magnetosonic modes, propagating normal to the magnetic field, grow exponentially and saturate at high values, due to the resistivity. We also demonstrate that Jeans-like instabilities can be enhanced inside a resistive fluid and that the formation of condensations influence the growing magnetosonic waves.
\end{abstract}

Key words. magnetic fields - magnetohydrodynamics (MHD) - instabilities - waves - cosmology: early Universe - relativity

\section{Introduction}

Magnetic fields are known to have a widespread presence in our Universe, being a common property of the intergalactic medium in galaxy clusters (Kronberg 1994). Reports on Faraday rotation imply significant magnetic fields in condensations at high redshifts (Kronberg et al. 1992). Large-scale magnetic fields and their potential implications for the formation and the evolution of the observed structures have been the subject of theoretical investigation (see Thorne 1967; Jacobs 1968; Ruzmaikina \& Ruzmaikin 1971; Wasserman 1978; Zel'dovich et al. 1983; Adams et al. 1996; Barrow et al. 1997; Tsagas \& Barrow 1997; Jedamzik et al. 1998; Barrow et al. 2006, etc.). Magnetic fields observed in galaxies and galaxy clusters are in energy equipartition with the gas and cosmic rays (Wolfe et al. 1992). The origin of these fields, which can be astrophysical, cosmological or both, remains an unresolved issue.

If magnetism has a cosmological origin, as observations of $\mu \mathrm{G}$ fields in galaxy clusters and high-redshift protogalaxies seem to suggest, it could have affected the evolution of the Universe (Giovannini 2004; Barrow et al. 2006). There are several scenarios for the generation of primordial magnetic fields (e.g. see Grasso \& Rubinstein 2001). Most of the early treatments were Newtonian, with relativistic studies making a recent appearance in the literature. A common factor between almost all the approaches is the use of the MHD approximation, namely the assumption that the magnetic field is frozen into an effectively infinitely conductive cosmic medium (i.e. a fluid of zero resistivity). With a few exceptions (e.g. see Fennelly 1980; Jedamzik et al. 2000; Vlahos et al. 2005), the role of kinetic viscosity and the possibility of non-zero resistivity have been ignored. Nevertheless, these aspects are essential for putting together a comprehensive picture of the magnetic behavior, particularly as regards the non-linear regime. The electric fields associated with the resistivity can become a source of particle acceleration, while the induced non-linear currents may react back on the magnetic field (Vlahos et al. 2005).

Many recent studies have used a Newtonian or a FriedmannRobertson-Walker (FRW) model to represent the evolving Universe and super-imposed a large-scale ordered magnetic field. The magnetic field is assumed to be too weak to destroy the FRW isotropy and the anisotropy, induced by it, is treated as a perturbation (Ruzmaikina \& Ruzmaikin 1971; Tsagas \& Barrow 1997; Durrer et al. 1998). Current observations give a strong motivation for the adoption of a FRW model but the uncertainties on the cosmological Standard Model are several. Therefore, the limits of the approximations and the effects one may lose by neglecting the anisotropy of the background magnetic field should be investigated. The formation of small-scale structures and the excitation of resistive instabilities in Bianchi-Type models have been explored (Fennelly 1980). Nevertheless, the excitation of MHD-waves in curved spacetime and their subsequent temporal evolution is far from being clearly understood (Papadopoulos et al. 2001).

In the present article we explore the evolution of a magnetized resistive plasma in an anisotropic cosmological model. We begin with a uniform plasma driving the dynamics of the curved spacetime (the so-called zeroth-order solution). This dynamical system is subsequently perturbed by small-scale fluctuations and we study their interaction with the anisotropic background, searching for imprints on the temporal evolution of the perturbations' amplitude.

In Sect. 2, we present the system of the field equations appropriate to describe the model under consideration. In Sect. 3, we solve this system analytically, to derive the zeroth-order solution. In Sect. 4, we extract the first-order perturbed equations. In Sect. 5, we derive the dispersion relation for the magnetized cosmological perturbations and in Sect. 6, we perform a numerical study of their evolution, using a fifth-order Runge-KuttaFehlberg temporal integration scheme. In Sect. 7, a perturbation 
analysis over purely gravitational fluctuations reveals an inherent Jeans-like instability.

Our results suggest that in a resistive plasma, within an interval of $10^{11} \mathrm{~s}$ after the beginning of the interaction process, fastmagnetosonic modes are excited, growing exponentially in time and saturate at high values. In this way, magnetic field perturbations can be retained at large amplitudes, for sufficiently long time-intervals $\left(\Delta t \sim 10^{12} \mathrm{~s}\right)$, resulting in the enhancement of the ambient magnetic field (dynamo effect). In addition, the resistive plasma enhances the condensations that can be formed within the anisotropic fluid due to gravitational instability, which, in turn, influences the growth of the magnetosonic waves.

\section{The field equations}

We consider an axisymmetric Bianchi-Type I cosmological model, driven by an anisotropic and resistive perfect fluid, in the presence of a time-dependent magnetic field, $\boldsymbol{B}=B(t) \hat{x}$. The corresponding line-element is written in the form

$\mathrm{d} s^{2}=-\mathrm{d} t^{2}+R^{2}(t) \mathrm{d} x^{2}+S^{2}(t)\left[\mathrm{d} y^{2}+\mathrm{d} z^{2}\right]$.

The evolution of a curved spacetime in the presence of matter and an e/m field is determined by the gravitational field equations

$\mathcal{R}_{\mu \nu}-\frac{1}{2} g_{\mu \nu} \mathcal{R}=+8 \pi G \mathcal{T}_{\mu \nu}$

(in the system of units where $\hbar=1=c$ ), together with the energy-momentum conservation law

$\mathcal{T}_{; v}^{\mu v}=0$

and Maxwell's equations

$F_{; v}^{\mu v}=4 \pi J^{\mu}$,

$F_{\mu v ; \lambda}+F_{\nu \lambda ; \mu}+F_{\lambda \mu ; \nu}=0$.

In Eqs. (2)-(5), Greek indices refer to the four-dimensional spacetime (Latin indices refer to the three-dimensional spatial section) and the semicolon denotes covariant derivative. $\mathcal{R}_{\mu v}$ and $\mathcal{R}$ are the Ricci tensor and the scalar curvature with respect to the background metric $g_{\mu \nu}$, while $G$ is Newton's gravitational constant. $F^{\mu \nu}$ is the antisymmetric tensor of the e/m field and $J^{\mu}$ is the corresponding current density.

The energy-momentum tensor consists of two parts

$\mathcal{T}^{\mu \nu}=\mathcal{T}_{\text {fluid }}^{\mu \nu}+\mathcal{T}_{\text {em }}^{\mu v}$.

The first part is due to an anisotropic perfect fluid source of the form

$\mathcal{T}_{\text {fluid }}^{\mu \nu}=\rho u^{0} u^{0}+p_{i} u^{i} u^{i}+p_{i} g^{i i}$

where $\rho(t)$ is the energy density, $p_{i}(t)$ are the components of the anisotropic pressure and the axial symmetry of the metric (1) implies that $p_{2}(t)=p_{3}(t) \cdot u^{\mu}=\mathrm{d} x^{\mu} / \mathrm{d} s$ is the fluid's fourvelocity, satisfying the conditions $u_{\mu} u^{\mu}=-1$ and $h^{\mu v} u_{\mu}=0$, with $h^{\mu \nu}=g^{\mu \nu}+u^{\mu} u^{v}$ being the projection tensor.

The second part is due to the ambient $\mathrm{e} / \mathrm{m}$ field

$\mathcal{T}_{\mathrm{em}}^{\mu \nu}=\frac{1}{4 \pi}\left(F^{\mu \alpha} F^{\nu \beta} g_{\alpha \beta}-\frac{1}{4} g^{\mu \nu} F_{\alpha \beta} F^{\alpha \beta}\right)$

where for $\boldsymbol{E}=\mathbf{0}$ and $\boldsymbol{B} / / \hat{x}$, the non-zero components of the Faraday tensor in the curved spacetime (1) read (see Appendix A)

$F^{23}=\frac{B^{x}}{S^{2}}=-F^{32}$.
The current density $J^{\mu}$ may be determined by the invariant form of Ohm's law

$J^{\mu}=n_{\mathrm{e}} e u^{\mu}+\frac{1}{\eta} F^{\mu v} u_{v}$

where $n_{\mathrm{e}}$ is the locally measured charge density and $\eta$ is the (finite) electric resistivity, in units of time. As a consequence of the Maxwell equations, we obtain $J_{; \mu}^{\mu}=0$. Assuming that that the fluid has zero net-charge, i.e. $n_{\mathrm{e}}=0$, Eq. (10) reduces to $J^{\mu}=\frac{1}{\eta} F^{\mu v} u_{v}$. A vanishing net charge indicates that the perfect fluid consists of at least two components.

\section{The background solution}

We look for axisymmetric Bianchi-Type I cosmological solutions to the Einstein-Maxwell equations (Appendices A and B), representing the background metric of our problem. In this case, Eqs. (2) reduce to

$2\left(\frac{\dot{R} \dot{S}}{R S}\right)+\left(\frac{\dot{S}}{S}\right)^{2}=8 \pi G \rho(t)+G B^{2}(t)$

$-2 \frac{\ddot{S}}{S}-\left(\frac{\dot{S}}{S}\right)^{2}=8 \pi G p_{1}(t)-G B^{2}(t)$

$-\left(\frac{\ddot{R}}{R}+\frac{\ddot{S}}{S}\right)-\left(\frac{\dot{R} \dot{S}}{R S}\right)=8 \pi G p_{2}(t)+G B^{2}(t)$

(the dot denotes time-derivative) and Eqs. (4), (5) yield

$\partial_{t}\left[S^{2} B(t)\right]=0$.

Equation (12) has a clear physical interpretation: the magnetic flux through a comoving surface normal to the direction of the magnetic field is conserved.

On the other hand, the continuity Eq. (3) results in (Appendix C)

$$
\begin{array}{r}
\partial_{t}\left[\rho(t)+\frac{B^{2}}{8 \pi}\right]+\frac{\dot{R}}{R}\left[p_{1}(t)-\frac{B^{2}}{8 \pi}\right]+2 \frac{\dot{S}}{S}\left[p_{2}(t)+\frac{B^{2}}{8 \pi}\right] \\
+\left(\frac{\dot{R}}{R}+2 \frac{\dot{S}}{S}\right)\left[\rho(t)+\frac{B^{2}}{8 \pi}\right]=0
\end{array}
$$

and the particles' number conservation law reads

$\dot{\rho}+\left(\frac{\dot{R}}{R}+2 \frac{\dot{S}}{S}\right) \rho=0$.

The system of Eqs. (11)-(14) admits the exact solution

$$
\begin{aligned}
& R(t)=\left(\frac{t}{t_{0}}\right), \quad S(t)=\left(\frac{t}{t_{0}}\right)^{\frac{1}{2}} \\
& \rho(t)=\rho_{0}\left(\frac{t_{0}}{t}\right)^{2} \quad B(t)=B_{0}\left(\frac{t_{0}}{t}\right) \\
& p_{1}(t)=p_{10}\left(\frac{t_{0}}{t}\right)^{2}, \quad p_{2}(t)=-p_{20}\left(\frac{t_{0}}{t}\right)^{2}
\end{aligned}
$$

where the index " 0 " stands for the corresponding values at $t=t_{0}$ and $t_{0}$ marks the beginning of the interaction between magnetized plasma and curved spacetime. Solution (15) represents an anisotropic cosmological model in which the large-scale anisotropy along the $\hat{x}$-axis is due to the presence of an ambient 
magnetic field. The combination of Eqs. (11) and (15) indicates that, initially, the total energy density is given by

$\rho_{0}+\frac{B_{0}^{2}}{8 \pi}=\frac{5}{32 \pi G t_{0}^{2}}$

and the difference between fluid's pressure and the pressure of the magnetic field along the two anisotropic spatial directions is equal

$p_{10}-\frac{B_{0}^{2}}{8 \pi}=\frac{1}{32 \pi G t_{0}^{2}}$

$p_{20}-\frac{B_{0}^{2}}{8 \pi}=\frac{1}{32 \pi G t_{0}^{2}}$.

Equations (17) lead us to identify

$p_{10}=p_{0}=p_{20}$

i.e. initially, when $R\left(t_{0}\right)=S\left(t_{0}\right)$, the two components of the anisotropic pressure were equal in absolute value, something that is confirmed also by Eq. (13). Furthermore, with Eqs. (16) and (17), we obtain

$p_{10}+2 p_{20}=\frac{3}{5}\left(\rho_{0}+6 \frac{B_{0}^{2}}{8 \pi}\right)$

which, according to Eq. (18), results in the equation of state for the matter-energy content at $t=t_{0}$

$p_{0}=\frac{1}{5}\left(\rho_{0}+6 \frac{B_{0}^{2}}{8 \pi}\right)$.

For $B_{0}=0$, i.e. as regards the perfect fluid itself, we obtain that, initially, $p_{0}=\frac{1}{5} \rho_{0}$. Since $p_{0}<\frac{1}{3} \rho_{0}$, our model corresponds to a semi-realistic cosmological model of Bianchi Type I. These models are crude, first order approximations to the actual Universe when we use currently available theories and observations (Jacobs 1969).

\section{The cosmological perturbations}

For any dynamical system, much can be learnt by investigating the possible modes of small-amplitude oscillations or waves. A plasma is physically much more complicated than an ideal gas, especially when there is an externally applied magnetic field. As a result, a variety of small-scale perturbations may appear. We first assume a uniform magnetized plasma in curved spacetime as background, which is perturbed by small scale fluctuations. In this article, the evolution of the background is described by the solution (15).

Accordingly, we introduce first-order perturbations in the Einstein-Maxwell equations, by decomposing the physical variables of the fluid as

$\rho(t, z)=\rho(t)+\delta \rho(t, z)$

$p_{x}(t, z)=p_{1}(t)$

$p_{y}(t, z)=p_{2}(t)-\delta p(t, z)$

$p_{z}(t, z)=p_{2}(t)+\delta p(t, z)$

and we insert the perturbed values (21) and (22) into Eqs. (11)-(14), neglecting all terms higher than or equal to the second order. The pressure perturbation $\delta p(t, z)$ introduces a longitudinal acoustic mode, propagating along the $\hat{z}$-direction and therefore

$\delta p(t, z)=C_{\mathrm{s}}^{2} \delta \rho(t, z)$

where $C_{\mathrm{s}}$ is the speed of sound. The four-velocity of the plasma fluid is perturbed around its comoving value, $u^{\mu}=(1,0,0,0)$, as

$u^{\mu}(t, z)=\left(1+\delta u^{0}(t, z), 0,0, \delta u^{z}(t, z)\right)$.

Then, the condition $u_{\mu} u^{\mu}=-1$, to the first leading order, implies

$\delta u^{0}(t, z)=0$

and, therefore, $u^{3}(t, z)=\delta u^{z}(t, z)$. Accordingly, $\rho(t, z) u^{3}(t, z)=$ $\rho(t) \delta u^{z}(t, z)+O_{2}$.

As regards the perturbations of the e/m field, we consider that they correspond to a transverse $\mathrm{e} / \mathrm{m}$ wave, propagating along the $\hat{z}$-axis $(\boldsymbol{k} / / \hat{z})$

$\boldsymbol{E}(t, z)=\delta E^{y}(t, z) \hat{y}$

$\boldsymbol{B}(t, z)=B(t) \hat{x}+\delta B^{x}(t, z) \hat{x}$.

Therefore, the non-zero components of the Faraday tensor in curved spacetime are modified as follows

$F^{02}=\frac{1}{S} \delta E^{y}(t, z)=-F^{20}$

$F^{23}=\frac{1}{S^{2}}\left[B(t)+\delta B^{x}(t, z)\right]=-F^{32}$.

In what follows, we take into account the so-called Cowling approximation (Cowling 1941), admitting that $\delta g_{\mu \nu}=0$. Therefore, the evolution of the perturbed quantities is governed only by the energy-momentum tensor conservation, together with Maxwell's equations.

To begin with, we perturb the particles' number conservation law: accordingly, Eq. (C.3) yields

$\partial_{t}(\delta \rho)+\rho(t) \partial_{z}\left(\delta u^{z}\right)+\delta \rho\left(\frac{\dot{R}}{R}+2 \frac{\dot{S}}{S}\right)=0$.

We continue with Maxwell's equations. Then, from Eq. (B.2), using Eqs. (21), (22), (24) and (25), we obtain

$$
\begin{array}{r}
-\partial_{t}\left(\delta E^{y}\right)+\frac{1}{S} \partial_{z}\left(\delta B^{x}\right)-\delta E^{y}\left(\frac{\dot{R}}{R}+\frac{\dot{S}}{S}\right)= \\
4 \pi \frac{1}{\eta}\left[\delta E^{y}+S B(t) \delta u^{z}\right] .
\end{array}
$$

Now, Eq. (B.3) becomes

$\partial_{t}\left(S^{2} \delta B^{x}\right)-S \partial_{z}\left(\delta E^{y}\right)=0$.

The conservation Eq. (C.2) results in

$\partial_{t}\left[\rho(t) \delta u^{z}-\frac{1}{4 \pi S} B(t) \delta E^{y}\right]+\frac{1}{S^{2}} \partial_{z}\left[\delta p+\frac{1}{4 \pi} B(t) \delta B^{x}\right]$
$+\left(\frac{\dot{R}}{R}+2 \frac{\dot{S}}{S}\right)\left[\rho(t) \delta u^{z}-\frac{1}{4 \pi S} B(t) \delta E^{y}\right]=0$

while, to the first leading order, Eq. (C.1) collapses to an identity. Equations (29)-(32) are the linearly independent first order perturbed Einstein-Maxwell equations in the curved background (1). In the flat spacetime - zero resistivity limit, they reduce to Eqs. (10.53a), (10.9) and (10.53c) of Jackson (1975), respectively. 
To develop the theory of small-amplitude waves in curved spacetime, we search for solutions to the linearized Eqs. (29)-(32) in which all perturbation quantities are proportional to the exponential

$\exp \left[\mathrm{i}\left(k z-\int^{t} \omega \mathrm{d} t\right)\right]$

following the so-called adiabatic approximation (Zel'dovich 1979; Birrell \& Davies 1982; Padmanabhan 1993). In this context, the (slowly varying) time-dependent frequency of the wave is defined by the eikonal

$\Omega=\int^{t} \omega \mathrm{d} t$

through the relation

$\omega=\frac{\mathrm{d} \Omega}{\mathrm{d} t}$.

Notice that, in Eq. (33), $z$ is the comoving coordinate along the $\hat{z}$-axis and $k$ is the comoving wave-number. In an expanding Universe, the corresponding physical quantities are defined as $z_{\mathrm{ph}}=z S(t)$ and $k_{\mathrm{ph}}=k / S(t)$, so that $k_{\mathrm{ph}} z_{\mathrm{ph}}=k z$.

\section{The dispersion relation}

Before discussing the temporal evolution of the perturbation quantities, it is important to trace what kind of waveforms are admitted by this system. We have to derive their dispersion relation, $D(k, \omega)=0$, at $t=t_{0}$. Provided that certain kinds of modes (such as acoustic, magnetosonic etc.) do exist, they can be excited through their interaction with the anisotropic spacetime. An additional excitation, due to the non-zero resistivity, is also possible (Fennelly 1980).

Accordingly, we assume a wave-like expansion for the perturbation quantities of the form

$\delta \rho=A_{\rho} \mathrm{e}^{\mathrm{i}\left(k z-\int^{t} \omega \mathrm{d} t\right)}, \quad \delta u^{z}=A_{u} \mathrm{e}^{\mathrm{i}\left(k z-\int^{t} \omega \mathrm{d} t\right)}$

$\delta E^{y}=A_{E} \mathrm{e}^{\mathrm{i}\left(k z-\int^{t} \omega \mathrm{d} t\right)}, \quad \delta B^{x}=A_{B} \mathrm{e}^{\mathrm{i}\left(k z-\int^{t} \omega \mathrm{d} t\right)}$

$\delta p=A_{p} \mathrm{e}^{\mathrm{i}\left(k z-\int^{t} \omega \mathrm{d} t\right)}=C_{s}^{2} A_{\rho} \mathrm{e}^{\mathrm{i}\left(k z-\int^{t} \omega \mathrm{d} t\right)}$.

Although the background quantities depend on time, in the search for a dispersion relation at $t=t_{0}$, we treat the perturbation amplitudes $\left(A_{i} \mathrm{~s}\right)$ as constants. In this way, our search for potential waveforms at $t=t_{0}$, is not disturbed by the inherent non-linearity introduced for $t>t_{0}$. Nevertheless, once the potential waveforms are determined, their interaction with the curved spacetime in the presence of an external magnetic field implies that for $t>t_{0}$ the time-dependence of their amplitudes is a priori expected. Using Eqs. (36), Eq. (29) is written in the form

$\left(H_{R}+2 H_{S}-\mathrm{i} \omega\right) \delta \rho=-\mathrm{i} k \rho(t) \delta u^{z}$

where we have set

$H_{R}=\frac{\dot{R}}{R}$ and $H_{S}=\frac{\dot{S}}{S}$.

Furthermore, using Eqs. (37), Eq. (30) reduce to

$\left[\mathrm{i} \omega-\left(H_{R}+H_{S}+\frac{4 \pi}{\eta}\right)\right] \delta E^{y}=-\mathrm{i} \frac{k}{S} \delta B^{x}+\frac{4 \pi}{\eta} S B(t) \delta u^{z}$ while Eq. (31) becomes

$\left(2 H_{S}-\mathrm{i} \omega\right) \delta B^{x}=\mathrm{i} \frac{k}{S} \delta E^{y}$.

Finally, Eq. (32) yields

$$
\begin{aligned}
& {\left[\dot{\rho}(t)+\left(H_{R}+2 H_{S}-\mathrm{i} \omega\right) \rho(t)\right] \delta u^{z}+\mathrm{i} \frac{k}{S^{2}} C_{\mathrm{s}}^{2} \delta \rho=} \\
& \frac{1}{4 \pi}\left[\frac{\mathrm{d}}{\mathrm{d} t}\left(\frac{B(t)}{S}\right)+\left(H_{R}+2 H_{S}-\mathrm{i} \omega\right) \frac{B(t)}{S}\right] \delta E^{y}-\mathrm{i} \frac{1}{4 \pi} k B(t) \delta B^{x}
\end{aligned}
$$

With the aid of Eqs. (12) and (14), the combination of Eqs. (39)-(43) results in

$$
\begin{aligned}
& {\left[-\omega^{2}+k_{\mathrm{ph}}^{2} C_{\mathrm{s}}^{2}-\mathrm{i} \omega\left(H_{R}+2 H_{S}\right)\right]} \\
& \quad \times\left[\left(\mathrm{i} \omega+H_{R}+H_{S}-\frac{4 \pi}{\eta}\right)\left(2 H_{S}-\mathrm{i} \omega\right)-k_{\mathrm{ph}}^{2}\right]= \\
& \quad \frac{4 \pi}{\eta} u_{\mathrm{A}}^{2}\left(H_{R}+2 H_{S}-\mathrm{i} \omega\right) \\
& \quad \times\left[\left(H_{R}-H_{S}-\mathrm{i} \omega\right)\left(2 H_{S}-\mathrm{i} \omega\right)+k_{\mathrm{ph}}^{2}\right]
\end{aligned}
$$

where $u_{\mathrm{A}}^{2}=\left(B_{0}^{2} / 4 \pi \rho_{0}\right)$ is the (dimensionless) Alfvén velocity. Equation (44) is the dispersion relation which determines the possible waveforms admitted by this dynamical system for all $t \geq t_{0}$.

$\omega$, as defined by Eqs. (34) and (35), has the usual meaning of the angular frequency of an oscillating process only in the shortwavelength (high-frequency) regime of the mode $k$ (Mukhanov et al. 1992). In other words, the wave description in curved spacetime makes sense only when the physical wavelength along the direction of propagation $\left[\lambda_{\mathrm{ph}}=\lambda S(t)\right]$ is much smaller than the corresponding horizon length $\left[\ell_{H_{S}}=H_{S}^{-1}(t)\right]$, i.e.

$\lambda_{\mathrm{ph}} \ll \ell_{H_{S}}$.

Equation (45) implies that, in the anisotropic background (1), the wave description makes sense as long as

$\omega, k_{\mathrm{ph}} \gg H_{R}, H_{S}$

for all $t \geq t_{0}$. In this limit, Eq. (44) becomes surprisingly transparent, namely

$\left(\omega^{2}-k_{\mathrm{ph}}^{2} C_{\mathrm{s}}^{2}\right)\left(\omega^{2}-k_{\mathrm{ph}}^{2}\right)+\mathrm{i} \omega \frac{4 \pi}{\eta}\left[\omega^{2}\left(1+u_{\mathrm{A}}^{2}\right)-k_{\mathrm{ph}}^{2}\left(C_{\mathrm{s}}^{2}+u_{\mathrm{A}}^{2}\right)\right]=0$.

The vanishing of the real part results in acoustic $\left(\omega=k_{\mathrm{ph}} C_{\mathrm{s}}\right)$ and $\mathrm{e} / \mathrm{m}\left(\omega=k_{\mathrm{ph}}\right)$ waves, while the vanishing of the imaginary part results in fast-magnetosonic waves

$\omega^{2}\left(1+u_{\mathrm{A}}^{2}\right)=k_{\mathrm{ph}}^{2}\left(C_{\mathrm{s}}^{2}+u_{\mathrm{A}}^{2}\right)$.

In the zero-resistivity limit (ideal plasma), the obvious modes expected are the magnetosonic modes, which we recover. On the other hand, in most astrophysical situations we have (Jackson 1975)

$u_{\mathrm{A}}^{2} \ll C_{\mathrm{s}}^{2}$.

In this case, Eq. (47) reads

$\left(\omega^{2}-k_{\mathrm{ph}}^{2} C_{\mathrm{s}}^{2}\right)\left(\omega^{2}+\mathrm{i} \frac{4 \pi}{\eta} \omega-k_{\mathrm{ph}}^{2}\right)=0$. 
According to Eq. (50), in the very high frequency limit where no acoustic waves are admitted, we are left with a waveform governed by the dispersion relation

$\omega^{2}+\mathrm{i} \frac{4 \pi}{\eta} \omega-k_{\mathrm{ph}}^{2}=0$

which yields

$\mathrm{e}^{-\mathrm{i} \int^{t} \omega \mathrm{d} t} \sim \mathrm{e}^{-\frac{2 \pi}{\eta} t}$

This result has a clear physical interpretation: all the very-highfrequency perturbations of the dynamical system are suppressed due to the finite resistivity. Therefore, the only modes that survive in a resistive cosmological model are the (low-frequency) MHD modes. In the next section, we discuss the evolution of these modes.

\section{Numerical study of the MHD mode}

In order to study the temporal evolution of the magnetosonic modes for $t \geq t_{0}$, we assume that their amplitudes are no longer time-independent

$\delta \rho=\tilde{\rho}(t) \mathrm{e}^{\mathrm{i}\left(k z-\int^{t} \omega \mathrm{d} t\right)}, \quad \delta u^{z}=\tilde{u}(t) \mathrm{e}^{\mathrm{i}\left(k z-\int^{t} \omega \mathrm{d} t\right)}$

$\delta E^{y}=\tilde{E}(t) \mathrm{e}^{\mathrm{i}\left(k z-\int^{t} \omega \mathrm{d} t\right)}, \delta B^{x}=\tilde{B}(t) \mathrm{e}^{\mathrm{i}\left(k z-\int^{t} \omega \mathrm{d} t\right)}$

$\delta p=\tilde{p}(t) \mathrm{e}^{\mathrm{i}\left(k z-\int^{t} \omega \mathrm{d} t\right)}=C_{\mathrm{s}}^{2} \tilde{\rho}(t) \mathrm{e}^{\mathrm{i}\left(k z-\int^{t} \omega \mathrm{d} t\right)}$.

In Eqs. (53)-(55), the wave-number $k$ is related to the frequency $\omega$ through Eq. (48) and, once again, we have taken into account the equation of state for the perfect fluid.

We decompose the time-dependent amplitude of the perturbations (53)-(55) into a real and an imaginary part, as

$$
\begin{aligned}
& \tilde{\rho}(t)=\rho_{R}(t)+\mathrm{i} \rho_{I}(t) \\
& \tilde{u}(t)=u_{R}(t)+\mathrm{i} u_{I}(t) \\
& \tilde{E}(t)=E_{R}(t)+\mathrm{i} E_{I}(t) \\
& \tilde{B}(t)=B_{R}(t)+\mathrm{i} B_{I}(t)
\end{aligned}
$$

that reduces Eqs. (29)-(32) to the following first order system

$$
\begin{gathered}
\dot{\rho}_{R}+\omega \rho_{I}-\rho_{0}\left(\frac{t_{0}}{t}\right)^{2} k u_{I}+\frac{2}{t} \rho_{R}=0 \\
\dot{\rho}_{I}-\omega \rho_{R}+\rho_{0}\left(\frac{t_{0}}{t}\right)^{2} k u_{R}+\frac{2}{t} \rho_{I}=0 \\
\dot{E}_{R}+\omega E_{I}+k\left(\frac{t_{0}}{t}\right)^{1 / 2} B_{I}+\frac{3}{2 t} E_{R}+4 \pi \frac{1}{\eta} E_{R} \\
+4 \pi \frac{1}{\eta} B_{0}\left(\frac{t_{0}}{t}\right)^{1 / 2} u_{R}=0 \\
\dot{E}_{I}-\omega E_{R}-k\left(\frac{t_{0}}{t}\right)^{1 / 2} B_{R}+\frac{3}{2 t} E_{I}+4 \pi \frac{1}{\eta} E_{I} \\
+4 \pi \frac{1}{\eta} B_{0}\left(\frac{t_{0}}{t}\right)^{1 / 2} u_{I}=0 \\
\dot{B}_{R}+\omega B_{I}+\frac{1}{t} B_{R}+k\left(\frac{t_{0}}{t}\right)^{1 / 2} E_{I}=0 \\
\dot{B}_{I}-\omega B_{R}+\frac{1}{t} B_{I}-k\left(\frac{t_{0}}{t}\right)^{1 / 2} E_{R}=0
\end{gathered}
$$

$$
\begin{gathered}
\rho_{0} \dot{u}_{R}+\omega \rho_{0} u_{I}+\left(\frac{1}{4 \pi t_{0}}+\frac{1}{\eta}\right) B_{0}\left(\frac{t_{0}}{t}\right)^{1 / 2} E_{R} \\
+\frac{1}{\eta} B_{0}^{2} u_{R}-k C_{s}^{2}\left(\frac{t}{t_{0}}\right) \rho_{I}=0 \\
\rho_{0} \dot{u}_{I}-\omega \rho_{0} u_{R}+\left(\frac{1}{4 \pi t_{0}}+\frac{1}{\eta}\right) B_{0}\left(\frac{t_{0}}{t}\right)^{1 / 2} E_{I} \\
+\frac{1}{\eta} B_{0}^{2} u_{I}+k C_{s}^{2}\left(\frac{t}{t_{0}}\right) \rho_{R}=0 .
\end{gathered}
$$

We integrate numerically the system (58)-(65), using a fifth order Runge-Kutta-Fehleberg scheme with variable integration step. The time is measured in units of $t_{0}$ and, therefore, $\tau=\frac{t}{t_{0}} \geq$ 1 . In terms of $\tau$, the physical wave-number reads $k_{\mathrm{ph}}=k / \sqrt{\tau}$ and the Hubble parameter along the $y z$-plane is written in the form $H_{S}=\left(2 \tau t_{0}\right)^{-1}$. According to Eq. (45), for a certain value of $\tau$, a wave is well inside the horizon as long as

$$
k \gg \frac{1}{2 \sqrt{\tau} t_{0}} \text {. }
$$

The validity of Eq. (66) for long $\tau$-intervals determines the appropriate values of the comoving wave-number. Now, the analysis depends on where do we place the initial time, $t_{0}$.

According to the Standard Model (Kolb \& Turner 1990), after nucleosynthesis the Universe goes on expanding and cooling until $t \sim 10^{13} \mathrm{~s}$. At that time, the temperature drops to the point where electrons and nuclei can form stable atoms (recombination). Before that time, during the so-called radiation epoch, photons couple strongly with matter, the main constituent of which is in the form of plasma. Therefore, the latest time at which plasma could play a role of cosmological significance is the recombination time $\left(t_{R}=1.2 \times 10^{13} \mathrm{~s}\right)$. In the limiting case where $t_{0}=t_{R}$, the condition (66) reads $k \gg \frac{1}{\sqrt{\tau}} \times 10^{-14} \mathrm{~s}^{-1}$ and, therefore, an appropriate choice for $k$ would be $k=10^{-12} \mathrm{~s}^{-1}$.

In order to decide on the initial values of the unperturbed quantities, we write Eq. (16) in ordinary units, namely

$\rho_{0} c^{2}+\frac{B_{0}^{2}}{8 \pi}=\frac{5 c^{2}}{32 \pi G t_{0}^{2}}$.

We adopt a typical behavior for the energy-density, valid at the late stages of the radiation epoch (see Weinberg 1972, Eq. (15.6.42))

$\rho_{0} c^{2}=1.45 \alpha T^{4}$

where $T$ is the temperature and $\alpha$ is the black-body constant. At the time of recombination $\left(t_{0}=1.2 \times 10^{13} \mathrm{~s}, T=4000 \mathrm{~K}\right)$, we obtain $\rho_{0} \equiv \rho_{0} c^{2}=2.8 \mathrm{erg} / \mathrm{cm}^{3}$, which, through Eq. (67), is effectively a choice on $B_{0}$, namely $B_{0} \simeq 7$ Gauss. Notice that this value lies barely within limits of the constraint

$\rho_{0} c^{2}>\frac{B_{0}^{2}}{8 \pi}$

a necessary condition to retain the anisotropy of the metric (Thorne 1967). Extrapolation of this result along the lines of Eq. (15) to the present epoch $\left(t_{\mathrm{p}} \simeq 15 \times 10^{9} y\right)$ suggests that, today, the corresponding magnetic field should be $B_{\mathrm{p}} \simeq 6.6 \times$ $10^{-10}$ Gauss. This value lies within limits of the upper bound for the present-day magnetic field strength, arising from the largeangular scale anisotropy of the microwave radiation background (MRB) at last scattering (Barrow et al. 1997, 2006)

$B<4 \times 10^{-9}$ Gauss. 
We estimate the amount of distortion which the expansion anisotropy along the $x$-axis (caused by the unperturbed magnetic field) induces on the microwave pattern at the present epoch. The contribution of a large-scale coherent magnetic field to the microwave quadrupole anisotropy is given by (Madsen 1989)

$\frac{\Delta T}{T} \simeq(1+z) \frac{B_{\mathrm{p}}^{2}}{8 \pi \epsilon_{\mathrm{p}}}$

where $B_{\mathrm{p}}$ and $\epsilon_{\mathrm{p}}$ denote the present values of the magnetic field and the background radiation energy-density, $z$ is the redshift at which the anisotropy begins to grow (in our case, at the recombination time where $z \simeq 1100$ ). The present value of the microwave background temperature is $T_{\mathrm{p}}=2.8 \mathrm{~K}$, corresponding to an energy-density of $\epsilon_{\mathrm{p}} \simeq 4.7 \times 10^{-13} \mathrm{erg} / \mathrm{cm}^{3}$ for the radiation field. Accordingly, our analysis suggests that the presentday quadrupole anisotropy along the $x$-axis should be

$\left.\frac{\Delta T}{T}\right|_{x} \simeq 4.06 \times 10^{-5}$

i.e. almost four times larger than the corresponding COBE result.

Taking into account that, initially, the unperturbed quantities are of the order of unity, we normalize all the perturbation quantities at $t=t_{0}$, to 0.01 in cgs units. On the other hand, initially, the equation of state for the perfect fluid admits $C_{\mathrm{s}}^{2}=0.2$, while, for the resistivity we adopt the Spitzer relation (Krall \& Trivielpiece 1973)

$\eta=10^{-2}\left(\frac{T}{\mathrm{eV}}\right)^{3 / 2} \mathrm{~s}$.

In a radiation-dominated background, we have (Kolb \& Turner 1990)

$\left(\frac{T}{\mathrm{eV}}\right)=\frac{10^{6}}{\sqrt{t(\mathrm{~s})}}$

and therefore, during recombination, Eq. (64) results in $\eta=$ $0.0645 \mathrm{~s}$. In order to demonstrate how $\eta$ may trigger instabilities, we consider three cases, namely $\eta=0.0645 \mathrm{~s}, \eta=0.0745 \mathrm{~s}$ and $\eta=0.0870 \mathrm{~s}$.

The output of the numerical integration consists of the electric and the magnetic field perturbations' amplitude

$$
\begin{aligned}
& \left|\delta E^{y}\right|=\sqrt{E_{R}^{2}+E_{I}^{2}} \\
& \left|\delta B^{x}\right|=\sqrt{B_{R}^{2}+B_{I}^{2}}
\end{aligned}
$$

and illustrates their temporal evolution. In Fig. 1, we present the magnetic field perturbation versus time. We consider two cases:

- For $\eta=0$ (ideal plasma), the magnetic field perturbation grows steeply at early times. It appears that the interaction of the perturbed quantities with the anisotropic spacetime results in the amplification of the convective field $\delta E_{1}^{y}=$ $-S B(t) \delta u^{z}$, which is the only one to survive in the idealplasma-limit [e.g. see Eq. (30)]. Through Faraday's law, any amplification in the convective field leads to an analogous growth in $\delta B^{x}$, at the expense of the cosmological expansion. Accordingly, after exhausting any available energy, the magnetic field perturbation reaches a maximum value before it is suppressed due to the cosmological redshift.

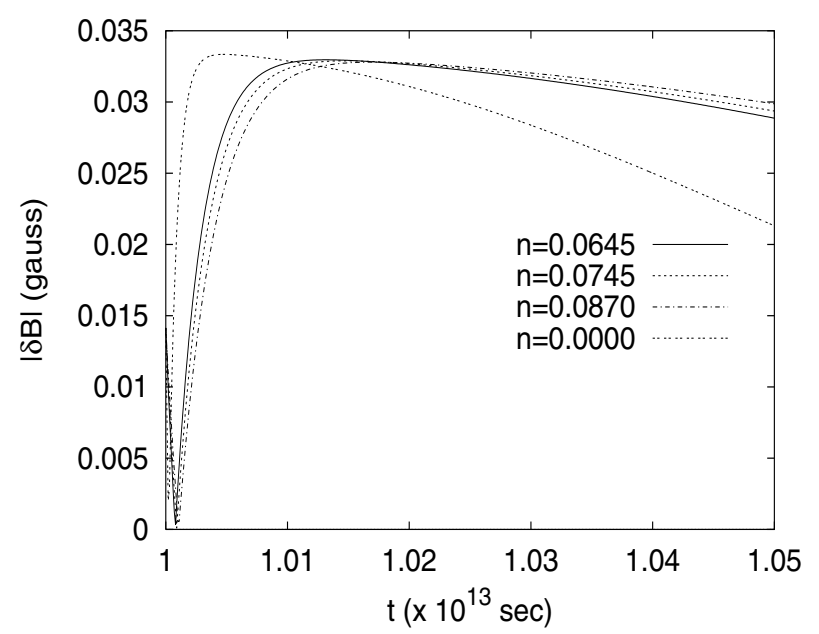

Fig. 1. The time-evolution of the magnetic field perturbation, for $B_{0}=$ 7 Gauss and for several values of the resistivity $\eta(\mathrm{s})$. Notice that, for $\eta \neq 0$, the perturbation's amplitude is saturated, acquiring large values for long enough time intervals.

- On the other hand, for $\eta \neq 0$, the magnetic field perturbation also increases rapidly at early times after $t_{0}\left(\Delta t \sim 10^{11} \mathrm{~s}\right)$, reaching values up to 3 times its initial one. However, in this case, the perturbation's amplitude is saturated, acquiring sufficiently large values for long enough time intervals $\left(\Delta t \sim 10^{12} \mathrm{~s}\right)$. This is due to the fact that, besides the convective field $\delta E_{1}^{y}$, a non-zero resistivity also favors convective currents $\left(\delta E_{2}^{y}=\eta \delta J^{y}\right)$. For $\eta \neq 0$, the lhs of Eq. (30) corresponds, through Ampere's law, to an electric current. Accordingly, now, the energy available to be absorbed by the perturbed quantities is larger and therefore the magnetic perturbation remains at high levels for longer time intervals. As a result, after saturation, the magnitude of $\delta B$ constitutes a fraction of $5 \times 10^{-3}$ of the unperturbed value of the magnetic strength. In this case, the quadrupole anisotropy induced in the MRB along the $x$-axis reads

$$
\left.\frac{\Delta T}{T}\right|_{x}(1+z) \frac{B^{2}}{8 \pi \epsilon} \simeq\left(1+2 \frac{\delta B}{B}\right)
$$

resulting in

$\left.\frac{\Delta T}{T}\right|_{x} \simeq 4.10 \times 10^{-5}$

i.e. the corresponding value is enlarged by $1 \%$.

The numerical results indicate a completely different behavior for the electric field perturbation (Fig. 2). Not only are the growth rate and the highest value of $\delta E^{y}$ slightly smaller than the corresponding values of $\delta B^{x}$, but, also, the suppression rate of the perturbation's amplitude is much larger than that of $\delta B^{x}$, resulting in a rapid decrease of the electric field at late times. It appears that the expanding Universe disfavors strong electric fields.

We conclude that, for reasonable values of the resistivity, the magnetic field perturbations lead to a real instability, acquiring large values for sufficiently long time-intervals. The influence of resistivity in triggering instabilities in anisotropic cosmological models has been the subject of research in the past (Fennelly 1980). To the best of our knowledge, however, this is the first time that a direct connection between the resistivity and the saturation of the perturbations' amplitude at high values for long time intervals is suggested and discussed. 


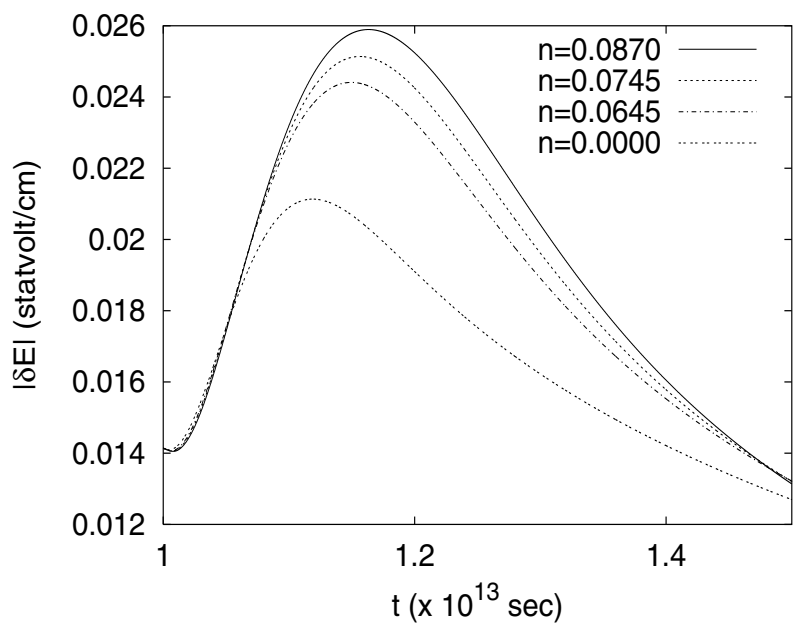

Fig. 2. The time-evolution of the electric field perturbation, for $B_{0}=$ 7 Gauss and for several values of the resistivity $\eta$ (s). Notice that, in this case, there is no saturation.

\section{Jeans-like instabilities}

The question that arises now is whether the cosmological model under consideration admits other kinds of instability and what their role is in connection to the resistive one. To answer this question, we study the evolution of purely gravitational perturbations, examining whether they admit a growing behavior (Jeans instability) or not.

In the absence of e/m fields (and their fluctuations), one is left with the system of perturbation equations

$\left(H_{R}+2 H_{S}-\mathrm{i} \omega\right) \delta \rho=-\mathrm{i} k \rho(t) \delta u^{z}$

$\left[\dot{\rho}(t)+\left(H_{R}+2 H_{S}-\mathrm{i} \omega\right) \rho(t)\right] \delta u^{z}=-\mathrm{i} \frac{k}{S^{2}} C_{s}^{2} \delta \rho$

the combination of which yields

$\left[\frac{\dot{\rho}}{\rho}+\left(H_{R}+2 H_{S}\right)-\mathrm{i} \omega\right]\left[\left(H_{R}+2 H_{S}\right)-\mathrm{i} \omega\right]=-\frac{k^{2}}{S^{2}} C_{\mathrm{s}}^{2}$.

Taking into account the particle number conservation law, Eq. (81) results in

$\omega^{2}+\mathrm{i} \omega\left(H_{R}+2 H_{S}\right)-k_{\mathrm{ph}}^{2} C_{\mathrm{s}}^{2}=0$

describing damped acoustic waves. With respect to $\omega$, Eq. (82) is a second order algebraic equation with roots

$\omega_{1,2}=-\mathrm{i} \frac{H_{R}+2 H_{S}}{2} \pm \sqrt{k_{\mathrm{ph}}^{2} C_{\mathrm{s}}^{2}-\left(\frac{H_{R}+2 H_{S}}{2}\right)^{2}}$

provided that

$k_{\mathrm{ph}} C_{\mathrm{s}} \geq \frac{H_{R}+2 H_{S}}{2}$.

In this case, the energy-density perturbations (36) reduce to

$\delta \rho=\frac{A_{\rho}}{S \sqrt{R}} \mathrm{e}^{\mathrm{i} k z \mp \mathrm{i} \int^{t} \omega_{R} \mathrm{~d} t}$

where $\omega_{R}$ is given by

$\omega_{R}^{2}=\frac{k^{2}}{S^{2}} C_{\mathrm{s}}^{2}-\left(\frac{H_{R}+2 H_{S}}{2}\right)^{2}$.

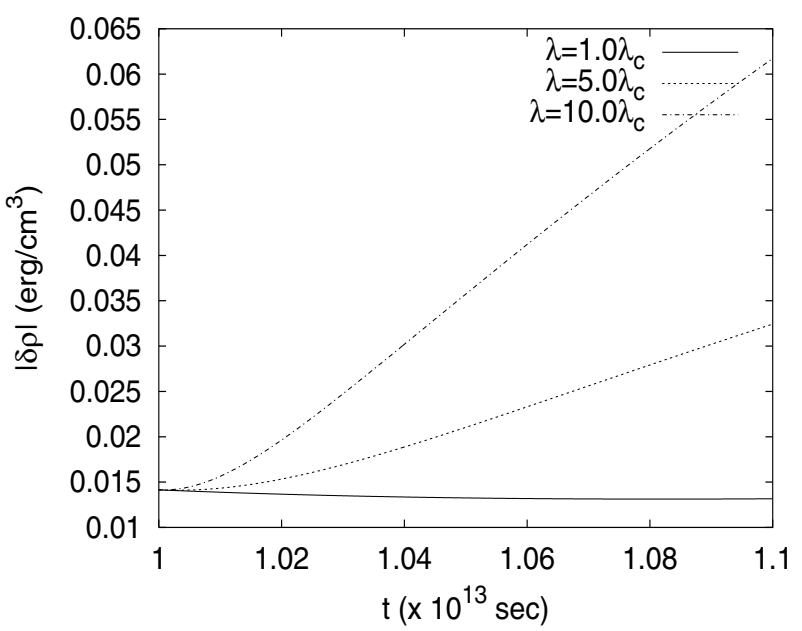

Fig. 3. The time-evolution of the energy density perturbation for several values of the coordinate wave-length $\lambda$ in terms of $\lambda_{\mathrm{c}}$.

Equation (86) represents the dispersion relation for the propagation of the energy-density fluctuations. In the isotropic case, where $H_{R}=H=H_{S}$, it yields

$\omega_{R}^{2}=\frac{k^{2}}{S^{2}} C_{\mathrm{s}}^{2}-\frac{9}{4} H^{2}$

which, with the aid of the corresponding Friedmann equation $H^{2}=\frac{8 \pi G}{3} \rho$, reads

$\omega_{R}^{2}=\frac{k^{2}}{S^{2}} C_{\mathrm{s}}^{2}-6 \pi G \rho$.

Equation (88) is identical to the isotropic (FRW) result, predicted by Weinberg (1972), in the relativistic theory of small fluctuations.

In contrast to the high frequency e/m waves (52), as regards the corresponding energy-density perturbations, propagation is possible only when their physical wave-number is larger than a characteristic value arising from condition (84) otherwise, after some time they become unstable and grow exponentially with time (Jeans-like instabilities).

Taking into account the background solution (15), Eq. (84) at $t=t_{0}$ reads

$k \geq k_{c}=\frac{1}{C_{\mathrm{s}} t_{0}}=1.86 \times 10^{-13} \mathrm{~cm}^{-1}$

and the corresponding Jeans length is given by

$\lambda_{\mathrm{c}}=2 \pi C_{\mathrm{s}} t_{0}=3.37 \times 10^{13} \mathrm{~cm}$.

Propagation of density perturbations with $\lambda>\lambda_{\mathrm{c}}$ is not possible, for all $t \geq t_{0}$ and we are lead to a gravitational instability. The larger the coordinate wave-length is, the more prominent the unstable behavior will be (Fig. 3).

Furthermore, one may ask whether the waves with wavenumber around $k_{\mathrm{c}}$ in a non-ideal plasma may grow faster than those in an ideal plasma. For every $t>t_{0}$, the physical Jeans length along the $\hat{x}$-axis $\left[\lambda_{x}=\lambda_{\mathrm{c}} R(t)\right]$ is larger than the corresponding length along the other two axes $\left[\lambda_{y}=\lambda_{\mathrm{c}} S(t)=\lambda_{z}\right]$, due to the background anisotropy, suggesting formation of "cigarlike" condensations within the anisotropic fluid. Since this fluid is conductive, these condensations act in favor of electric currents which may lead to a further amplification of the e/m perturbations, fortifying any pre-existing resistive instability (Fig. 4). 


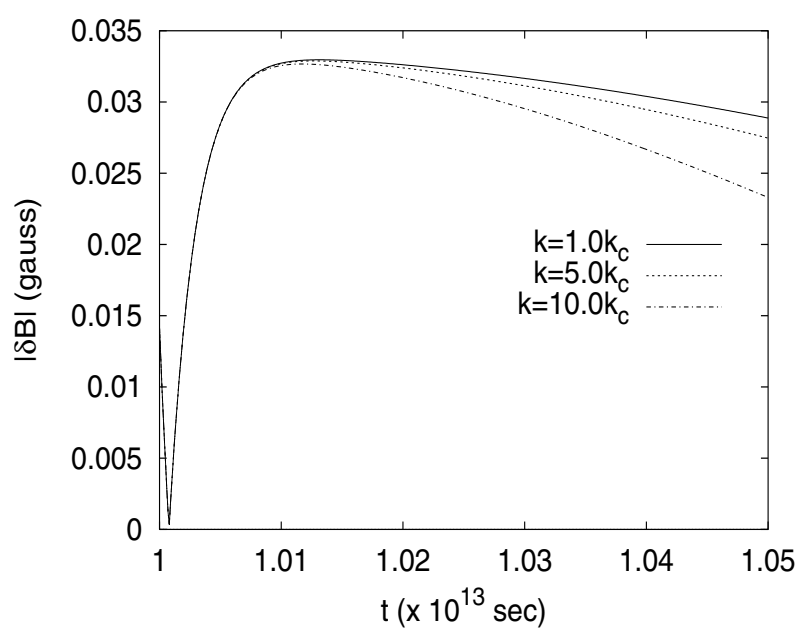

Fig. 4. The time-evolution of the magnetic field perturbation for $\eta=$ $0.0645 \mathrm{~s}, B_{0}=7$ Gauss and for several values of the coordinate wavenumber in terms of $k_{\mathrm{c}}$.

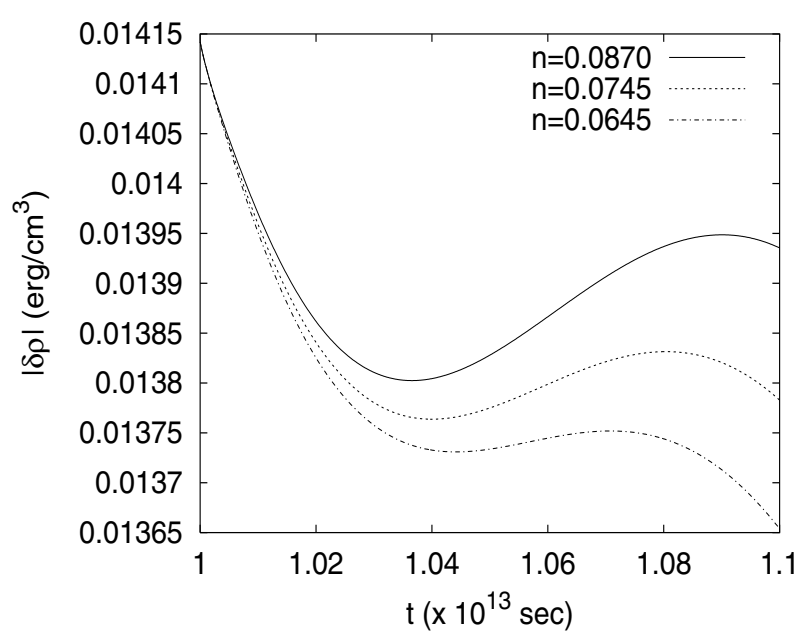

Fig. 5. The time-evolution of the energy density perturbation, for $k=k_{\mathrm{c}}$ and for several values of the resistivity $\eta$ (s). We observe that the Jeans instability becomes more prominent as the resistivity grows.

Therefore, a Jeans-like instability enhances the phenomena related to the resistivity.

On the other hand, numerical results indicate that waves with wavelength around $k_{\mathrm{c}}$ become more prominent as the resistivity grows (Fig. 5). This result also has a clear physical interpretation: as we have already seen, any increase in the resistivity fortifies the surrounding magnetic field. A strong magnetic field organizes plasma along its lines, favoring any pre-existing condensations. Hence, resistive instabilities act in favor of the corresponding gravitational ones and vice versa.

\section{Discussion}

We study the evolution of the magnetosonic waves in a magnetized, resistive plasma, which governs the dynamics of an anisotropic cosmological model. After constructing the general set of MHD and Einstein equations for the anisotropic cosmological model (see the Appendices), we solve the field equations to obtain the zeroth-order solution. In order to determine the waveforms admitted by this system in the first place, we introduce wave-like perturbations and, neglecting all terms higher or equal than the second order, we extract the dispersion relation at $t=t_{0}$, i.e. at the beginning of the interaction between magnetized plasma and curved spacetime. It appears that magnetosonic modes can be excited due to the anisotropy and the resistivity. For $t \geq t_{0}$, we integrate numerically the perturbed equations, using the dispersion relation for the fast-magnetosonic waves.

We find that, at early times, both the electric and the magnetic field perturbations grow exponentially, at least in the regime where the linear analysis holds. However there is a major difference in their behavior in the presence of a non-zero resistivity. For $\eta \neq 0$, the magnetic field perturbation after increasing to reach values up to 3 times its initial one, is subsequently saturated, remaining at high levels for sufficiently long time intervals $\left(\Delta t \sim 10^{12} \mathrm{~s}\right)$.

The situation is completely different to the electric field perturbation. Not only the growth rate and the highest value of $\delta E^{y}$ are slightly smaller than the corresponding values of $\delta B^{x}$, but, also, the suppression rate of the perturbation's amplitude is much larger than that of $\delta B^{x}$. Accordingly, the electric field decreases rapidly at late times. It appears that the expanding Universe disfavors strong electric fields.

We have shown that waves with wave-number around $k_{\mathrm{c}}$ are enhanced in non ideal plasmas.

Acknowledgements. The authors would like to thank Dr. Heinz Ishliker and Dr. Christos Tsagas for helpful discussions. Financial support from the Greek Ministry of Education under the Pythagoras Program, is gratefully acknowledged.

\section{Appendix A}

We present the closed set of MHD and Einstein equations (in the system of units where $\hbar=1=c$ ) for the anisotropic cosmological models of Bianchi-Type I

$\mathrm{d} s^{2}=-\mathrm{d} t^{2}+R^{2}(t, z) \mathrm{d} x^{2}+S^{2}(t, z) \mathrm{d} y^{2}+T^{2}(t, z) \mathrm{d} z^{2}$

in the presence of an anisotropic perfect fluid, which allows for acoustic waves along the $\hat{z}$-direction

$\mathcal{T}_{\text {fluid }}^{\mu \nu}=\rho u^{\mu} u^{v}+p_{i} u^{i} u^{i}+p_{i} g^{i i}$

and an e/m field of the form

$F^{\hat{\alpha} \hat{\beta}}=\left[\begin{array}{cccc}0 & 0 & E^{y} & 0 \\ 0 & 0 & 0 & 0 \\ -E^{y} & 0 & 0 & B^{x} \\ 0 & 0 & -B^{x} & 0\end{array}\right]$

where Greek indices refer to the four-dimensional spacetime and Latin indices refer to the three-dimensional spatial section. In Eq. (A.3), $F^{\hat{\alpha} \hat{\beta}}$ is the Faraday tensor in flat spacetime. The components of the e/m field in curved spacetime are defined by

$F^{\mu v}=F^{\hat{\alpha} \hat{\beta}} e_{\hat{\alpha}}^{\mu} e_{\hat{\beta}}^{v}$

where the non-zero components of the orthonormal tetrad $e_{\hat{\alpha}}^{\mu}$ of the local Lorentz frame for the metric (A.1) are given by

$e_{\hat{t}}^{\mu}=(-1,0,0,0) \quad e_{\hat{x}}^{\mu}=\left(0, \frac{1}{R}, 0,0\right)$

$e_{\hat{y}}^{\mu}=\left(0,0, \frac{1}{S}, 0\right) \quad e_{\hat{z}}^{\mu}=\left(0,0,0, \frac{1}{T}\right)$.

Therefore, in the curved spacetime (A.1), the non-zero components of the Faraday tensor are

$F^{02}=\frac{E^{y}}{S}=-F^{20}$
$F^{23}=\frac{B^{x}}{S T}=-F^{32}$. 
In what follows, the dot denotes the time-derivative, while the prime denotes differentiation with respect to $z$. The Einstein equations, $G_{\mu \nu}=8 \pi G\left(\mathcal{T}_{\mu \nu}^{\text {fluid }}+\mathcal{T}_{\mu \nu}^{\mathrm{em}}\right)$, result in:

The $(t t)$-component is given by

$$
\begin{aligned}
& -\frac{1}{T^{2}}\left(\frac{R^{\prime \prime}}{R}+\frac{S^{\prime \prime}}{S}\right)+\left(\frac{\dot{R} \dot{S}}{R S}+\frac{\dot{S} \dot{T}}{S T}+\frac{\dot{T} \dot{R}}{T R}\right) \\
& -\frac{1}{T^{2}}\left(\frac{R^{\prime} S^{\prime}}{R S}+\frac{S^{\prime} T^{\prime}}{S T}+\frac{T^{\prime} R^{\prime}}{T R}\right)=8 \pi G \rho+G\left[\left(E^{y}\right)^{2}+\left(B^{x}\right)^{2}\right] .
\end{aligned}
$$

The $(x x)$-component is given by

$$
\begin{array}{r}
-R^{2}\left(\frac{\ddot{S}}{S}+\frac{\ddot{T}}{T}\right)+\frac{R^{2}}{T^{2}}\left(\frac{S^{\prime \prime}}{S}\right)-R^{2}\left(\frac{\dot{S} \dot{T}}{S T}\right)+\frac{R^{2}}{T^{2}}\left(\frac{T^{\prime} S^{\prime}}{T S}\right)= \\
8 \pi G R^{2} p_{x}-G R^{2}\left[-\left(E^{y}\right)^{2}+\left(B^{x}\right)^{2}\right] .
\end{array}
$$

The (yy)-component is given by

$$
\begin{array}{r}
-S^{2}\left(\frac{\ddot{R}}{R}+\frac{\ddot{T}}{T}\right)+\frac{S^{2}}{T^{2}}\left(\frac{R^{\prime \prime}}{R}\right)-S^{2}\left(\frac{\dot{R} \dot{T}}{R T}\right)-\frac{S^{2}}{T^{2}}\left(\frac{T^{\prime} R^{\prime}}{T R}\right)= \\
8 \pi G S^{2} p_{y}+G S^{2}\left[-\left(E^{y}\right)^{2}+\left(B^{x}\right)^{2}\right] .
\end{array}
$$

The (zz)-component is given by

$$
\begin{array}{r}
-T^{2}\left(\frac{\ddot{S}}{S}+\frac{\ddot{R}}{R}\right)+\left(\frac{T^{\prime \prime}}{T}\right)-T^{2}\left(\frac{\dot{S} \dot{R}}{R S}\right)-\left(\frac{R^{\prime} S^{\prime}}{R S}\right)= \\
8 \pi G T^{2} p_{z}+G T^{2}\left[+\left(E^{y}\right)^{2}+\left(B^{x}\right)^{2}\right] .
\end{array}
$$

The $(t z)$-component is given by

$$
\left(\frac{\dot{R}^{\prime}}{R}+\frac{\dot{S}^{\prime}}{S}-\frac{R^{\prime} \dot{T}}{R T}-\frac{S^{\prime} \dot{T}}{S T}\right)=-8 \pi G T^{2} \rho u^{z}+2 G T E^{y} B^{x} .
$$

\section{Appendix B}

The Maxwell equations in curved spacetime are written in the form

$F_{; \beta}^{\alpha \beta}=F_{, \beta}^{\alpha \beta}+\Gamma_{\mu \beta}^{\beta} F^{\alpha \mu}=4 \pi J^{\alpha}$
$F_{\alpha \beta ; \gamma}+F_{\beta \gamma ; \alpha}+F_{\gamma \alpha \cdot \beta}=0$

where $J^{\alpha}=\frac{1}{\eta} F^{\alpha \beta} u_{\beta}$ is the current density and $\eta$ is the electric resistivity of the fluid. Accordingly, we obtain

$$
\begin{aligned}
-\partial_{t} E^{y}+\partial_{z}\left(\frac{B^{x}}{T}\right)-E^{y}\left(\frac{\dot{R}}{R}+\frac{\dot{T}}{T}\right) \\
+\frac{B^{x}}{T}\left(\frac{R^{\prime}}{R}+\frac{S^{\prime}}{S}+\frac{T^{\prime}}{T}\right)=4 \pi \frac{1}{\eta}\left(u^{0} E^{y}+T B^{x} u^{z}\right)
\end{aligned}
$$

and

$$
-\partial_{z}\left(S E^{y}\right)+\partial_{t}\left(S T B^{x}\right)=0 .
$$

\section{Appendix C}

Taking the time and space component of $T_{; v}^{\mu \nu}=0$, we obtain the required equations of motion in a covariant form, namely

$$
\begin{aligned}
& \partial_{t}\left[\rho(t)+\frac{1}{8 \pi}\left(E^{2}+B^{2}\right)\right]+\partial_{z}\left[\rho u^{z}-\frac{1}{4 \pi T} E B\right] \\
& +\frac{\dot{R}}{R}\left[p_{1}(t)-\frac{1}{8 \pi}\left(-E^{2}+B^{2}\right)\right]+\frac{\dot{S}}{S}\left[p_{2}(t)+\frac{1}{8 \pi}\left(-E^{2}+B^{2}\right)\right] \\
& \quad+\frac{\dot{T}}{T}\left[p_{3}(t)+\frac{1}{8 \pi}\left(+E^{2}+B^{2}\right)\right] \\
& \quad+\left(\frac{\dot{R}}{R}+\frac{\dot{S}}{S}+\frac{\dot{T}}{T}\right)\left[\rho(t)+\frac{1}{8 \pi}\left(E^{2}+B^{2}\right)\right]=0
\end{aligned}
$$

and

$$
\begin{aligned}
\partial_{t}\left[\rho u^{z}-\frac{1}{4 \pi T} E B\right]+\partial_{z}\left\{\frac{1}{T^{2}}\left[p_{3}(t)+\frac{1}{8 \pi}\left(E^{2}+B^{2}\right)\right]\right\} \\
\quad-\frac{R^{\prime}}{R T^{2}}\left[p_{1}(t)-\frac{1}{8 \pi}\left(-E^{2}+B^{2}\right)\right] \\
\quad-\frac{S^{\prime}}{S T^{2}}\left[p_{2}(t)+\frac{1}{8 \pi}\left(-E^{2}+B^{2}\right)\right] \\
+\frac{T^{\prime}}{T^{3}}\left[p_{3}(t)+\frac{1}{8 \pi}\left(E^{2}+B^{2}\right)\right] \\
+\left(\frac{\dot{R}}{R}+\frac{\dot{S}}{S}+\frac{\dot{T}}{T}\right)\left[\rho u^{z}-\frac{1}{4 \pi T} E B\right] \\
\quad+\left(\frac{R^{\prime}}{R}+\frac{S^{\prime}}{S}+\frac{T^{\prime}}{T}\right) \frac{1}{T^{2}}\left[p_{3}(t)+\frac{1}{8 \pi}\left(E^{2}+B^{2}\right)\right]=0 .
\end{aligned}
$$

In addition, the particles' number conservation law $\left(\rho u^{\mu}\right)_{; \mu}=0$, results in

$$
\begin{aligned}
& \partial_{t}\left(\rho u^{0}\right)+\partial_{z}\left(\rho u^{z}\right) \\
& \quad+\rho u^{0}\left(\frac{\dot{R}}{R}+\frac{\dot{S}}{S}+\frac{\dot{T}}{T}\right)+\rho u^{z}\left(\frac{R^{\prime}}{R}+\frac{S^{\prime}}{S}+\frac{T^{\prime}}{T}\right)=0 .
\end{aligned}
$$

\section{References}

Adams, J., Danielsson, U. H., \& Rubinstein, H. 1996, Phys. Lett. B, 388, 253 Barrow, J. D., Ferreira, P. G., \& Silk, J. 1997, Phys. Rev. Lett., 78, 3610 Barrow, J. D., Maartens, R., \& Tsagas, C. G. 2006, Cosmology with inhomogeneous magnetic fields [arXiv:astro-ph/0611537]

Birrell, N. D., \& Davies, P. C. W. 1982, Quantum Fields in Curved Space (Cambridge: Cambridge University Press)

Cowling, T. G. 1941, MNRAS, 101, 367

Durrer, R., Kahniashvil, T., \& Yates, A. 1998, Phys. Rev. D, 58, 123004

Fennelly, A. J. 1980, Phys. Rev. Lett., 44, 955

Giovannini, M. 2004, Int. J. Mod. Phys. D, 13, 391

Grasso, D., \& Rubinstein, H. 2001, Phys. Rep., 348, 163

Jackson, J. D. 1975, Classical Electrodynamics (New York: Wiley and Sons)

Jacobs, K. C. 1968, ApJ, 153, 661

Jacobs, K. C. 1969, ApJ, 155, 379

Jedamzik, K., Katalinic, V., \& Olinto, A. 1998, Phys. Rev. D, 57, 3264

Jedamzik, K., Katalinic, V., \& Olinto, A. 2000, Phys. Rev. Lett., 85, 700

Kolb, E. W., \& Turner, M. S. 1990, The Early Universe (Redwood California: Addison-Wesley)

Krall, N. A., \& Trivielpiece, A. W. 1973, Principles of Plasma Physics (New York: Mc Graw Hill)

Kronberg, P. P. 1994, Rep. Prog. Phys., 57, 325

Kronberg, P. P., Perry, J. J., \& Zukowski, E. L. H. 1992, ApJ, 387, 528

Madsen, M. S. 1989, MNRAS, 237, 109

Mukhanov, V. F., Feldman, H. A., \& Brandenberger, R. H. 1992, Phys. Rep., 215, 203

Padmanabhan, T. 1993, Structure Formation in the Universe (Cambridge: Cambridge University Press)

Papadopoulos, D., \& Esposito, F. P. 1982, ApJ, 257, 10

Papadopoulos, D. B., Vlahos, L., \& Esposito, F. P. 2001, A\&A, 382, 1

Ruzmaikina, T. V., \& Ruzmaikin, A. A. 1971, Sov. Astron., 14, 963

Thorne, K. S. 1967, ApJ, 148, 51

Tsagas, C. G., \& Barrow, J. D. 1997, Class. Quantum. Grav., 14, 2539

Vlahos, L., Tsagas, C. G., \& Papadopoulos, D. B. 2005, ApJ, Ł9

Wasserman, I. 1978, ApJ, 224, 337

Weinberg, S. 1972, Gravitation and Cosmology (New York: Wiley and Sons)

Wolfe, A. M., Lanzetta, K. M., \& Oren, A. L. 1992, ApJ, 388, 17

Zel'dovich, Ya. B. 1979, Creation of particles by the gravitational field, in Physics of the Expanding Universe, ed. M. Demianski (New York: Springer Verlag)

Zel'dovich, Ya. B., Ruzmaikin, A. A., \& Sokoloff, D. D. 1983, Magnetic Fields in Astrophysics (New York: Gordon and Breach) 